\title{
CORONARY HEART DISEASE IN WOMEN: A CHALLENGE FOR THE 21ST CENTURY
}

\author{
Maria Cecília Solimene
}

doi: $10.1590 / \mathbf{S 1 8 0 7 - 5 9 3 2 2 0 1 0 0 0 0 1 0 0 0 1 5}$

Solimene MC. Coronary heart disease in women: a challenge for the $21^{\text {st }}$ century. Clinics. 2010; 65(1):99-106.

Heart disease is the first killer of women in the modern era, regardless of age, race and of ethnicity, although its prevalence rises after menopause. Modern women have professional and housewife responsibilities, consume excess of fat and carbohydrates, smoke, do not exercise regularly and do not have enough time to rest. This situation leads to overweight, dyslipidemia, arterial hypertension, impaired glucose tolerance and diabetes. Women do not often participate in preventive studies and still undergo less intensive and invasive evaluation and treatment for chest pain when compared to men. However, the rate of coronary death is twice higher in women than in men after myocardial infarction and revascularization procedures. The objective of this review is to analyze the main gender differences regarding symptoms, diagnosis, management and prognosis of coronary heart disease and to discuss the influence of hormonal replacement therapy in the prevention of cardiovascular disease in postmenopausal women.

KEYWORDS: Women; Coronary disease; Diagnosis; Prognosis; Hormone therapy.

Over several decades there has been a general belief that women should not have coronary heart disease and exceptionally would experience a heart attack. Nowadays it is recognized that this belief it is not true, as heart disease is the first killer of women, regardless of race and of ethnicity; it also strikes at younger ages than most people think and the risk rises in the middle age; mostly, two-thirds of women who have heart attacks never fully recover. ${ }^{1}$ In 2006, 315,000 American women died from heart disease, in contrast with 82,000 deaths from stroke and 41,000 from breast cancer. ${ }^{1}$ In Brazil, 69,493 people died from myocardial infarction in $2006,40.8 \%$ of whom were women. ${ }^{2}$ Women in the modern era have many professional responsibilities, in addition to their responsibility to take care of their home, children, husband and older relatives. On the other hand, more and more women live by themselves. In general, women consume an excess of fat and carbohydrates, do not exercise regularly and do not have enough

Department of Clinical Cardiology, Instituto do Coração (InCor), Hospital das Clínicas da Faculdade de Medicina da Universidade de São Paulo - São Paulo/SP, Brazil.

Email: maria.solimene@incor.usp.br

Tel.: 55113069.5352

Received for publication on October 08, 2009.

Accepted for publication on October 26, 2009. time to rest. This situation leads to overweight, dyslipidemia, arterial hypertension, impaired glucose tolerance and diabetes. Most importantly, smoking is increasing among women. It is well known that diabetes and smoking are strong predictors of coronary events in both genders, but the risk is two to four-fold greater in women. ${ }^{3}$ Diabetes in women is frequently associated with myocardial infarction, heart failure and death. ${ }^{3}$ In the past, there was a lack of participation of women in preventive and research studies and there is increasing evidence that women undergo intensive and invasive evaluation and treatment for chest pain much less frequently than men. When acute coronary events occur in women, they are generally older, have multiple risk factors and coexisting illnesses and the rate of in-hospital death is always substantially greater than in men, even after revascularization procedures. ${ }^{3}$ Women and men differ in symptoms, diagnostic procedures and prognosis of coronary heart disease. These differences will be discussed in this review.

\section{Chest pain in women}

Chest pain has consistently been underestimated in women because of the disappointing results of evaluations of this symptom in the past. In the CASS (Coronary Artery 
Surgery Study), 30\% of women with typical angina and 64\% with atypical angina had normal coronary angiograms, but this was observed in only $7 \%$ and $34 \%$ of men, respectively. ${ }^{4}$ Syndrome $\mathrm{X}$, which was defined as symptoms and signs of myocardial ischemia in the presence normal coronary angiograms, ${ }^{5}$ predominates in women, but this syndrome may represent microvascular disease or endothelial dysfunction, which are more often observed in women. Recently, data from WISE (Women's Ischemia Syndrome Evaluation) and WTH (Women Take Heart) demonstrated that rates of cardiovascular events were highest for symptomatic women with nonobstructive coronary artery disease compared to symptomatic women with normal coronary arteries; on the other hand, symptomatic women with normal coronary arteries had almost three-fold higher rates of events when compared to asymptomatic women. ${ }^{6}$ These facts suggest that in women, as in men, chest pain compatible with angina deserves careful evaluation.

\section{Silent myocardial ischemia in women}

Silent myocardial ischemia, the objective evidence of myocardial ischemia in the absence of angina or anginal equivalents, is considered more prevalent in men than in women. In the ACIP (Asymptomatic Cardiac Ischemia Pilot) study the diagnosis of silent ischemia was difficult in women because ambulatory electrocardiographic monitoring failed to demonstrate ischemia in daily activities, and women with positive exercise testing did not have significant obstructive coronary lesions at angiography; for this reason, women represented only $25 \%$ of the studied population. ${ }^{7}$ Patients of both sexes with silent myocardial ischemia usually have severe and extensive coronary artery disease ${ }^{8}$ and unfavorable prognosis. ${ }^{9}$ However, totally asymptomatic women are still considered at low risk for coronary disease unless they have diabetes or peripheral arterial disease. ${ }^{10}$

\section{Noninvasive diagnosis of ischemia in women}

The standard exercise electrocardiogram testing is the most commonly used of the noninvasive tests for the assessment of myocardial ischemia. However, electrocardiographic changes during exercise are considered of diminished accuracy in women (sensitivity of approximately $60 \%$, specificity of approximately $70 \%$ ) due in part to an increased rate of false positive results. ${ }^{10}$ Several factors may influence exercise testing responses in women, such as a lower prevalence of coronary artery significant lesions; a higher prevalence of single vessel disease; microvascular disease and vascular spasms; limited exercise tolerance; breast attenuation artifacts; hormonal influences mimicking digitalis-like false positive responses.
Anatomical factors, such as smaller coronary artery size and smaller left ventricular chamber size and metabolic and hemodynamic influences, such as a lesser increase in the left ventricular ejection fraction and an inappropriate release of catecholamines. ${ }^{11}$ Many investigators have attempted to improve the diagnostic accuracy of exercise testing in women by creating new variables and formulas. One of these is the DTS (Duke Treadmill Score), which considers exercise time, ST deviation and effort angina; although the DTS has been related to the probability of severe coronary disease and survival, ${ }^{12}$ it has not found widespread acceptance in clinical practice. The exercise or pharmacological stress-imaging methods are now preferred as initial strategies for intermediate to high-risk women; however, the exercise electrocardiographic test has a high negative predictive value in women at low risk. ${ }^{10}$

According to the American Heart Association, women are considered to be at intermediate or high-risk for coronary heart disease if they have typical or atypical angina and are over 50 years, or are younger than 50 years but have typical angina; asymptomatic women at any age are considered of low-risk unless they are diabetic or present peripheral artery disease. ${ }^{10}$

\section{Myocardial perfusion imaging}

Cardiovascular imaging may be obtained by myocardial scintigraphy or echocardiography. Perfusion imaging with exercise or pharmacological stress has provided important information on the risk stratification of men and women; however, this technique has some limitations in women due to attenuation of myocardial activity caused by the presence of the breasts and because of the smaller female heart. These problems were mostly encountered with tallium-201 scintigraphy, but they were almost eliminated with the use of $99 \mathrm{~m}$-Tc-sestamibi. ${ }^{10}$ Taillefer et al. ${ }^{13}$ reported that the specificity for coronary lesions of $\geq 70 \%$ was $67.2 \%$ for Tl-201, 84.4\% for Tc-99m sestamibi SPECT perfusion and $92.2 \%$ for Tc-99m sestamibi-gated SPECT. On the other hand, both Tl-201 and Tc-99m sestamibi had similar sensitivities for the detection of ischemia. ${ }^{13}$ Santana-Boado et al. ${ }^{14}$ analyzed 702 consecutive men and women (44\%) submitted to exercise myocardial perfusion imaging using Tc-99m-MIBI and reported that the sensitivity in women was significantly lower than in men ( $85 \%$ vs. $93 \%$, respectively) but that the specificities were similar $(91 \%$ vs. $96 \%$ respectively) for those who underwent coronary angiography. After correction for the patients without coronary angiography, the sensitivity and specificity were not significantly different in men and women and the authors suggested that the diagnostic accuracy of Tc-99m-MIBI was globally satisfactory. ${ }^{14}$ Berman et al. ${ }^{15}$ reported relevant prognostic information with adenosine myocardial perfusion 
SPECT; they followed 2,656 women and 2,677 men over $27.0 \pm 8.8$ months and observed annual rates of cardiac death of $5.5 \%$ and $7.0 \%$, respectively, in men and women with severe abnormal scans. In diabetic patients, the predicted rate of cardiac death in diabetic women with severe abnormal scans was $8.5 \%$, which is significantly higher than the $6.0 \%$ predicted mortality for diabetic men with severe imaging abnormalities; among non-diabetics, the predicted survival was similar in men and women. ${ }^{15}$ Pharmacological stress imaging is indicated in patients with a poor tolerance to exercise, such as older women, obese patients, patients with a left bundle-branch block and diabetic women.

Stress Echocardiography provides valuable information about ventricular function and stress-induced ischemia. Exercise echocardiography may be performed via a treadmill or supine or upright bicycle exercise; pharmacological stress may be performed using a vasodilator (dipyridamole or adenosine) or dobutamine-atropine. Marwick et al. ${ }^{16}$ compared the results of exercise testing with those of exercise echocardiography in women the sensitivities of both tests were similar, $77 \%$ and $81 \%$, respectively, but the specificity and accuracy of exercise echocardiography were significantly higher than those of exercise testing, $80 \%$ vs. $56 \%$ and $81 \%$ vs. $64 \%$, respectively. ${ }^{16}$ Similar results were observed when exercise testing was compared to high-dose dipyridamoleechocardiography in women; the sensitivities were similar, $69 \%$ and $77 \%$, respectively, but the specificity and accuracy of dipyridamole-echocardiography were significantly higher than those of exercise testing, $93 \%$ vs. $52 \%$ and $87 \%$ vs. $59 \%$, respectively. ${ }^{17}$ On the other hand, according to a report from Dionisopoulos et al., ${ }^{18}$ dobutamine-stress echocardiography has demonstrated high sensitivity, specificity and accuracy in both men and women: $85 \%, 96 \%$ and $88 \%$ and $90 \%, 79 \%$ and $86 \%$, respectively, for men and women. Pharmacological stress echocardiography with dobutamine seems to provide better sensitivity than with dipyridamole; therefore, dobutamine is more commonly used. ${ }^{10}$ Tsutsui et al. ${ }^{19}$ reported gender differences in chronotropic and hemodynamic responses during dobutamine-atropine echocardiography; among patients who were not being treated with beta-blockers or calcium channel blockers, the heart rate response was higher in women than in men, the test duration was shorter in women and the total doses of dobutamine and atropine used during the tests were lower in women; no serious adverse effects were observed.

\section{Perfusion imaging or echocardiography in women?}

Shaw et al..$^{20}$ recently compared the prognostic value of stress echocardiography versus SPECT imaging. The authors analyzed data for 7,397 women submitted to stress echocardiography and 13,039 women submitted to SPECT imaging and concluded that both methods have a similar predictive value of provocative ischemia in estimating cardiac death and non-fatal myocardial infarction. ${ }^{20}$ When imaging tests detect ischemia in intermediate to high-risk women, coronary angiography is indicated.

\section{Myocardial infarction in women}

Myocardial infarction in women deserves special attention because women have worse in-hospital and longterm prognosis than men. It is generally recognized that women have a two-fold higher mortality after myocardial infarction compared to men. ${ }^{3}$ In the pre-thrombolytic era, 30 -day mortality rates up to $28 \%$ among women and $16 \%$ among men were reported and the rates of reinfarction were three-fold higher in women than in men. ${ }^{21}$ The highest mortality rates occurred for anterior wall myocardial infarction and among the elderly; however, there was a consistent 2:1 death ratio when women were compared to men. ${ }^{3}$ Thrombolytic therapy after myocardial infarction dramatically reduced mortality rates after myocardial infarction, but women still died twice as often as men. The Global Utilization of Streptokinase and Tissue Plasminogen Activator for Occluded Coronary Arteries (GUSTO-I) trial $^{22}$ analyzed the mortality and clinical and angiographic characteristics in 543 women and 1887 men; the unadjusted 30-day mortality rate after myocardial infarction was $13.1 \%$ in women, which is significantly higher than the $4.8 \%$ rate for men. The mortality rate of women with a patent infarctrelated artery was $11.5 \%$ versus $3.8 \%$ for men, while the mortality rate of women with an occluded infarct-related artery was $19 \%$ versus $5.4 \%$ for men; all of these differences were significant. ${ }^{22}$ The women enrolled in GUSTO-I were significantly older than the men and had more hypertension, diabetes and hypercholesteromia; after adjustment for age and clinical and angiographic variables, gender remained an independent predictor of 30-day mortality. ${ }^{22}$

In 1998, a report of the Third International Study of Infarct Survival (ISIS-3) Collaborative Group ${ }^{23}$ led to different conclusions; data obtained from 9,600 women and 26,480 men with myocardial infarction reported 35day mortality rates of $14.8 \%$ and $9.1 \%$, respectively. The mortality rate was significantly higher in females; however, when women and men were compared within three similarly sized groups ( $<60,60$ to 69 , and $\geq 70$ years), the mortality rate differences were reduced with decreasing age. ${ }^{23}$ Although the early mortality rate was about twice as high in women as in men, after the adjustment for age and other prognostic factors, gender had at most a small independent effect on early mortality after myocardial infarction..$^{23}$ 
The following year, Vaccarino et al. ${ }^{24}$ analyzed data on 384,878 patients $(155,565$ women) enrolled in the National Registry of Myocardial Infarction (NRMI) between June 1984 and January 1998; the overall mortality rate during hospitalization was $16.7 \%$ among women and $11.1 \%$ among men; the sex-based differences in rates varied according to age; among patients less than 50 years of age, the mortality rate for women was more than twice that for the men; the difference decreased as age increased and was no longer significant after the age of $74 .{ }^{24}$ In 2001, MacIntyre et al. ${ }^{25}$ reported data on all National Health Service hospital admissions in Scotland. Among 201,114 patients $(87,833$ women) admitted between 1986 and 1995, gender-based differences in survival varied according to age, with younger women (less than 55 years of age) having a significantly higher 30 -day mortality rate $(6.5 \%)$ when compared to age-matched men (4.8\%); with increasing age, the disparity in the 30-day survival was attenuated and even reversed in favor of women aged over 75 years. ${ }^{25}$

In our Institution, Conti et al. ${ }^{6}$ analyzed 236 consecutive young patients (54 women) with acute myocardial infarction, aged from 27 to 45 years, and observed that the reinfarction rate was three-fold higher in women than in men during hospitalization; the mortality rate did not differ significantly, but was higher in women (5.5\%) than in men $3.3 \%$ ). Recently, the Acute Myocardial Infarction in Switzerland (AMIS) Plus Registry ${ }^{27}$ analyzed 5,633 women and 14,657 men with acute coronary syndromes (ACS), 60\% with ST-segment elevation (STE) myocardial infarction. The mortality rate was $13.0 \%$ in women with STE-ACS and $7.5 \%$ in women with non-STE-ACS; these rates were significantly higher than the respective mortality rates in men, $7.2 \%$ and $4.9 \% .{ }^{27}$ The gender differences in the in-hospital mortality were mostly due to younger age of patients: the in-hospital mortality according to age group showed that significantly more women than men died only among the group aged less than 50 years. These findings challenge the general belief that age but not gender is responsible for the excess mortality of women after myocardial infarction.

\section{Why do young women have unfavorable outcomes after myocardial infarction?}

This question remains to be answered, but several hypotheses exist.Young women with coronary heart disease may be particularly predisposed to have more aggressive disease that may override the protective effect of estrogens. ${ }^{24}$ Young women with coronary heart disease may have more risk factors, but adjustments for diabetes and other risk factors accounted for only about $10 \%$ of the gender differences in the NRMI. Young women may have a hypercoagulable state, coronary spasms or microvascular disease; plaque erosions are predominant in young women who die suddenly while older women present rupture of plaques; also, young women have less coronary narrowing than older women and men. ${ }^{24}$ These and other mechanisms may be genetic in nature or have a genetic predisposition. The complete explanation of the unfavorable prognosis of young women with myocardial infarction is far from elucidated.

\section{Myocardial revascularization in women}

Coronary artery bypass grafts $(\mathrm{CABG})$ have been performed for several decades and have been shown to relieve angina and ischemia and prolong survival in selected groups, such as patients with left main coronary artery disease or three-vessel disease and ventricular dysfunction. However, the in-hospital mortality after CABG has always been reported as twice as high in women relative to men. ${ }^{3}$ This increase has been attributed to anatomical and clinical differences: women are generally older; have a smaller body size and coronary lumen; and have a higher incidence of risk factors, such as diabetes, systemic hypertension ${ }^{3}$ and hypercholesteromia. In 1993, Rahimtoola et al. ${ }^{28}$ published the results of $\mathrm{CABG}$ for 1,979 women and 6,927 men: the operative mortality was found to be $2.7 \%$ for women and $1.9 \%$ for men; women had a lower long-term survival compared to men in a 15 to 18 -year follow-up. When all of the risk factors and patient characteristics were considered, the independent risk factors for poorer survival were older age, previous $\mathrm{CABG}$, previous myocardial infarction and diabetes, but not gender. ${ }^{28}$

In 2002, Vaccarino et al. ${ }^{29}$ studied 51,187 patients $(15,178$ women) included in the National Cardiovascular Network database; in all age groups, women had less severe coronary artery disease and higher left ventricular ejection when compared to men. In the youngest age category $(<50$ years old), the in-hospital mortality rate was three-fold higher for women compared to men (3.4\% vs. $1.1 \%)$; among the patients aged 50-69 years, women were 2.4-fold more likely to die compared to men $(2.6 \%$ vs. $1.1 \%)$; the genderbased differences decreased with increasing age. ${ }^{29}$ These findings were similar when gender differences in mortality were analyzed after myocardial infarction. More recently, Humphries et al. ${ }^{30}$ reported data from the British Columbia Cardiac Registry demonstrating a significant improvement in short-term mortality in women undergoing CABG from 1991 to 2004. The authors analyzed 20,229 men and 4,983 women and observed that the 30-day mortality decreased significantly in men $(2.4 \%$ to $1.9 \%)$ and women $(5.6 \%$ to 
$1.9 \%)$ over the 14-year study period; overall, the 30-day mortality was $2.0 \%$ in men and $3.6 \%$ in women, and the greatest difference was observed in the $<50$-year-old group. ${ }^{30}$ There is no definite explanation for these divergent results. Besides the genetic and vascular mechanisms that have been discussed above, coronary disease is not very frequent in women under 50 years. It is likely that only the most severe cases are recognized and receive $\mathrm{CABG} .{ }^{29}$ On the other hand, Puskas et al. ${ }^{31}$ reviewed data for 42,477 consecutive patients (11,785 women) submitted to CABG in 63 North American centers during a two-year period (2004-2005); onpump CABG (ONCAB) was compared to off-pump CABG (OPCAB). After an adjustment for preoperative risk factors, patients treated with OPCAB had a significantly reduced risk of death, stroke, myocardial infarction and other adverse outcomes when compared to those treated with ONCAB; the benefits were most apparent in women. ${ }^{31}$ The authors concluded that OPCABG reduced the gender disparity in clinical outcomes after coronary revascularization. ${ }^{31}$

Percutaneous coronary interventions in women are less successful when compared to men. The National Heart Lung and Blood Institute (NHLBI) investigators ${ }^{32}$ analyzed the results of percutaneous transluminal coronary angioplasty (PTCA) performed during 1985/1986 in 2,136 patients (546 women); women were older than men, had more coronary risk factors and worse anginal symptoms. There was no gender difference when successful procedures were considered; however, the in-hospital mortality was significantly higher in women as compared to men, $2.6 \%$ and $0.3 \%$, respectively. ${ }^{32}$ The mortality rate among women older than 65 years was $5.6 \%$, which is ten-fold higher than that for men. ${ }^{32}$ These results led some physicians o contraindicate angioplasty in women aged 65 or older. ${ }^{33}$ Later, another report of the NHLBI experience in 1993/1994 demonstrated better clinical outcomes including survival in women submitted to PTCA, although the women were older than those analyzed in 1985/1986 and had multiple co-morbidities. ${ }^{34}$

A later report from the Bypass Angioplasty Revascularization Investigation (BARI) group analyzed 489 women and 1340 men and showed no gender differences in early or late mortality after PTCA and CABG. ${ }^{35}$ These results have not been confirmed by other investigators; Vakili et al. ${ }^{36}$ conducted a retrospective cohort study of all patients undergoing primary PTCA for a first acute myocardial infarction in New York state in 1995. 1,044 patients (317 women) were identified; the women were older than the men and had significantly more risk factors. The men were treated earlier, with a shorter time following symptom onset (within 6 hours), compared to women. ${ }^{36}$ In this analysis, the unadjusted in-hospital mortality rate was significantly higher in women $(7.9 \%)$ than in men $(2.3 \%)$; after adjustment for clinical variables and risk factors, women maintained a 2.3-fold higher risk of in-hospital death compared to men. ${ }^{36}$ Watanabe et al. ${ }^{37}$ analyzed data obtained from the Nationwide Inpatient Sample in 1997; during the study period, 118,548 PTCA were performed and 59\% involved the placement of stents. Analysis were performed separately for patients with and without acute myocardial infarction. ${ }^{37}$ Women had a two-fold higher mortality rate than men despite the presence of myocardial infarction, this gender difference remained significant for patients who underwent conventional PTCA or stenting. ${ }^{37}$ In this study, female gender was an independent predictor of death. ${ }^{37}$ In the recent report of the Acute Myocardial Infarction in Switzerland (AMIS) Plus Registry, ${ }^{27}$ the in-hospital mortality of women who underwent percutaneous coronary intervention was significantly higher than that of men $(4.2 \%$ vs. $3.0 \%$, respectively); the trend of higher mortality was observed among women younger than 50 years. In summary, women still have a worse prognosis than men when submitted to myocardial revascularization procedures.

\section{Why are there gender differences in coronary heart disease outcomes?}

This question also remains to be answered. Women may differ from men in several aspects: a) at the molecular level, sex-gene related diseases may occur; b) physiological differences may exist due to sex-hormones and to different responses to risk factors causing different patterns of illness; c) different responses to pharmacological agents may occur, due to pharmacokinetic differences, e.g. a different metabolization rates, side-effects and risks; d) the level of disability may be mediated by sex-specific disease expression. Coronary heart disease in women deserves further investigation and is a challenge that should be faced in the $21^{\text {st }}$ century.

\section{Hormonal replacement therapy and cardiovascular risk}

Previous observational epidemiological studies have suggested that hormone replacement therapy could reduce morbidity and mortality from coronary heart disease in women after menopause; however, the incidence of endometrial cancer, breast cancer, stroke and venous thromboembolism could be increased. ${ }^{38}$ In 1995, the Postmenopausal Estrogen/Progestin Interventions (PEPI) trial enrolled 875 healthy postmenopausal women aged 45 to 64 years who had no known contraindication to hormone therapy. ${ }^{39}$ The participants were randomly assigned in equal numbers to the following groups: 1) placebo; 2) conjugated 
equine estrogen (CEE), $0.625 \mathrm{mg} / \mathrm{d}$; 3) CEE, $0.625 \mathrm{mg} / \mathrm{d}$ plus cyclic medroxyprogesterone acetate (MPA), $10 \mathrm{mg} / \mathrm{d}$ for $12 \mathrm{~d} / \mathrm{mo}$; 4) CEE, $0.625 \mathrm{mg} / \mathrm{d}$ plus consecutive MPA, $2.5 \mathrm{mg} / \mathrm{d}$; or 5) CEE, $0.625 \mathrm{mg} / \mathrm{d}$ plus cyclic micronized progesterone (MP), $200 \mathrm{mg} / \mathrm{d}$ for $12 \mathrm{~d} / \mathrm{mo}^{39}$ After a 3 -year follow up, estrogen alone or in combination with a progestin improved lipoprotein levels (increased HDL-C and decreased LDL-C) and lowered fibrinogen plasma levels without detectable effects on the post-challenge insulin level or blood pressure. ${ }^{39}$ In women with a uterus, CEE plus cyclic MP had the most favorable effect on HDL-C and was associated with no excess risk of endometrial hyperplasia. ${ }^{39}$ After publication of the beneficial results of PEPI, the Heart and Estrogen/progestin Replacement Study (HERS),${ }^{40}$ a randomized clinical trial, was conducted briefly, 2,763 postmenopausal women younger than 80 years (mean age 66.7 years) with established coronary disease who had not had a hysterectomy were enrolled. The women were randomly assigned to the placebo or CEE+MPA daily group; after a 4-year follow-up, the hormone replacement therapy did not reduce coronary events, but increased the rate of thromboembolism and gall bladder disease. ${ }^{40}$ Based on these findings, hormone replacement therapy was not recommended for secondary prevention of coronary heart disease. The results were disappointing. The HERS was criticized because the enrolled women were old and had previous coronary disease. A subsequent study, the Women's Health Initiative (WHI), ${ }^{41,42}$ was conducted involving 27,347 postmenopausal women who were to be followed for eight to nine years; the women were aged 50 to 79 years, with $( \pm 10 \%)$ and without coronary disease, with a uterus or with a prior hysterectomy. The first arm of WHI enrolled 16,608 postmenopausal women with a uterus, with (minority) and without coronary disease and a mean age of 63.2 years; they were randomly assigned to the placebo or CEE+MPA daily group; after a mean of 5.2 years of follow-up, the study was interrupted because hormone therapy was associated with a $29 \%$ increase in coronary events, $26 \%$ increase in breast cancer, $41 \%$ increase in stroke and a twofold increase in thromboembolism. ${ }^{41}$ The second arm of WHI enrolled 10,739 postmenopausal women with prior hysterectomy, with (minority) and without coronary disease and a mean age of 63.6 years; they were randomly assigned to the placebo or CEE+MPA group. ${ }^{42}$ After a mean of 6,8 years of follow-up, the study was interrupted because hormone therapy was associated with a $39 \%$ increase of stroke; notably the risk of coronary events was not affected. ${ }^{42}$ The disappointing results of WHI led the investigators to a secondary analysis of their data; they studied hormone therapy and the risk of cardiovascular disease by age and years after menopause. ${ }^{43}$ The analysis of this study was based on combined data from the two trials: separate tests for trends were performed to examine differences in the hormone effects across three preselected, coded categories of age (50-59, 60-69, 70-79 years) or years since menopause $(<10,10-19, \geq 20){ }^{43}$ The numbers of events increased with increasing age; there were no significant increases in risks due to hormone therapy for coronary disease and death at ages 50 to 59 years, although the risk of stroke was increased at all ages. ${ }^{43}$ The risk of coronary events and death did not increase in women that initiated hormone therapy with less than 10 years since menopause, but increased significantly in women with 20 or more years after menopause. ${ }^{43}$ The risk of stroke was increased at any time. ${ }^{43}$ The WHI investigators concluded that hormone therapy could be used short-term for the relief of moderate to severe vasomotor symptoms, but not to prevent cardiovascular disease. ${ }^{43}$ At present, the American Heart Association ${ }^{44}$ and the European Society of Cardiology ${ }^{45}$ agree that hormone replacement therapy cannot be recommended for the prevention of cardiovascular disease in postmenopausal women.

In conclusion, women do have coronary heart disease and die twice as often as men after myocardial infarction and myocardial revascularization procedures. The recommendations for prevention of mortality consist of major traditional risk factor interventions addressing blood pressure, glucose and lipid plasma levels and lifestyle interventions, including smoking cessation, increased physical activity and weight control. Hormone replacement therapy is contraindicated for the prevention of cardiovascular disease in postmenopausal women. Women deserve careful evaluation for coronary symptoms even though some diagnostic methods are less accurate in women than in men. Women are less frequently treated for coronary disease as compared to men. We hope that such gender differences in the management of coronary disease will decrease in the $21^{\text {st }}$ century.

\section{REFERENCES}

1. The Heart Truth. A National Awareness Campaign for Women about Heart Disease, National Heart, Lung and Blood Institute. Available at www.nhlbi.nhi.gov/health/hearttruth.

2. Datasus: banco de dados. Avaliable at http://www.datasus.gov.br.
3. Da Luz PL, Solimene MC. Peculiaridades da doença arterial coronária na mulher. Rev Ass Med. Bras. 1999;45:45-54.

4. Chaitman BR, Bourassa MG, Davis K, Rogers W, Tyras DH, Berger R, et al. Angiographic prevalence of high-risk coronary artery disease in patients subsets (CASS). Circulation. 1981;64:360-7. 
5. Solimene MC, Lage SG, Ramires JAF. Insuficiência coronária em pacientes com coronárias normais. A persistência de um desafio. Arq. Bras. Cardiol. 1993;60:265-8.

6. Gulati M, Cooper-DeHoff RM, McClure C, Johnson BD, Shaw LJ, Handberg EM, et al. Adverse cardiovascular outcomes in women with nonobstructive coronary artery disease. A report from the Women's Ischemia Syndrome Evaluation Study and the St James Women Take Heart Project. Arch Int Med. 2009;169:843-50.

7. Frishman WH, Gomberg-Mattland M, Hirsch H, Catanese J, FuriaPalazzo S, Mueller H, et al. Differences between male and female patients with regard to baseline demographics and clinical outcomes in the Asymptomatic Cardiac Ischemia Pilot (ACIP) Trial. Clin Cardiol. 1998;21:184-90.

8. Sharaf BL, Williams DO, Miele NJ, McMahon RP, Stone PH, Bjerregaard $\mathrm{P}$, et al. A detailed angiographic analysis of patients with ambulatory electrocardiographic ischemia: results from the Asymptomatic Cardiac Ischemia Pilot (ACIP) Study angiographic core laboratory. J Am Coll Cardiol. 1997;29:78-84.

9. Solimene MC, Ramires JAF. Isquemia miocárdica assintomática. In Sousa AGMR, Mansur AJ. editores. SOCESP Cardiologia. vol.2. São Paulo: Editora Atheneu, 1996, p.417-25.

10. Mieres JH, Shaw LJ, Arai A, Budoff MJ, Flamm SD, Hundley G, et al. Role of noninvasive testing in the clinical evaluation of women with suspected coronary artery disease. Consensus statement from the Cardiac Imaging Committee, Council on Clinical Cardiology, and the Cardiovascular Imaging and Intervention Committee, Council on Cardiovascular Radiology and Intervention, American Heart Association. Circulation. 2005;111:682-96.

11. Stangl V, Witzel V, Baumann G, Stangl K. Current diagnostic concepts to detect coronary artery disease in women. Eur Heart J. 2008; 29;707-17.

12. Alexander KP, Shaw LJ, Delong ER, Mark DB, Peterson ED. Value of exercise treadmill testing in women. J Am Coll Cardiol. 1998;32:1657-64.

13. Taillefer R, DePuey EG, Udelson JE, Beller GA, Latour Y, Reeves F. Comparative diagnostic accuracy of Tl-201 and Tc-99m sestamibi SPECT imaging (perfusion and ECG-gated SPECT) in detecting coronary artery disease in women. J Am Coll Cardiol. 1997;29:69-77.

14. Santana-Boado C, Candell-Riera J, Castell-Conesa J, Aguadé-Bruix S, García-Burillo A, Canela T, et al. Diagnostic accuracy of technetium99m-MIBI myocardial SPECT in women and men. J Nucl Med. 1998;751-5.

15. Berman DS, Kang X, Hayes SW, Friedman JD, Cohen I, Abidov A, et al. Adenosine myocardial perfusion single-photon emission computed tomography in women compared with men. Impact of diabetes mellitus on incremental prognostic value and effect on patient management. J Am Coll Cardiol. 2003;41;1125-33.

16. Marwick TH, Anderson T, Williams J, Haluska B, Melin JA, Pashkow $\mathrm{F}$, et al. Exercise echocardiography is an accurate and cost-efficient technique for detection of coronary artery disease in women. J Am Coll Cardiol. 1995;26:335-41.

17. Masini M, Picano E, Lattanzi F, Distante A, L’Abbate A. High dose dipyridamole-echocardiography test in women: correlation with exercise-electrocardiographic test and coronary arteriography. J Am Coll Cardiol. 1988;12:682-5.
18. Dionisopoulos PN, Collins JD, Smart SC, Knickelbine TA, Sagar KB. The value of dobutamine stress echocardiography for the detection of coronary artery disease in women. J Am Soc Echocardiogr. 1997;10:811-17.

19. Tsutsui JM, Falcão SNRS, Dourado PMM, Lima MF, Alves AA, Guerra VC, et al. Gender differences in chronotropic and hemodynamic responses during dobutamine-atropine stress echocardiography. Echocardiography. 2007;24:843-50.

20. Shaw LJ, Vasey C, Sawada S, Rimmerman C, Marwick TH. Impact of gender on risk stratification by exercise and dobutamine stress echocardiography: long-term mortality in 4234 women and $6898 \mathrm{men}$. Eur Heart J. 2005;26:447-56.

21. Kannel WB, Sorlie P, McNamara PM. Prognosis after initial myocardial infarction: the Framingham study. Am J Cardiol. 1979;44:53-9.

22. Woodfield SL, Lundergan CF, Reiner JS, Thompson MA, Rohrbeck $\mathrm{SC}$, Deychak Y, et al. Gender and acute myocardial infarction: is there a different response to thrombolysis? J Am Coll Cardiol. 1997;29:35-42.

23. Malacrida R, Genoni M, Maggioni AP, Spataro V, Parish S, Palmer A, et al. A comparison of the early outcome of acute myocardial infarction in women and men. The Third International Study of Infarct Survival Collaborative Group. N Engl J Med. 1998;338:8-14.

24. Vaccarino V, Parsons L, Every NR, Barron HV, Krumholz HM Sex-based differences in early mortality after myocardial infarction. National Registry of Myocardial Infarction 2 Participants. N Engl J Med. 1999;341:217-25

25. McIntire K, Stewart S, Capewell C, Chalmers JWT, Pell JP, Boyd J, et al. Gender and survival: a population-based study of 201,114 men and women following a first acute myocardial infarction. J Am Coll Cardiol. 2001;38:729-35.

26. Conti RAS, Solimene MC, Da Luz PL, Benjó AM, Lemos Neto PA, Ramires JA. Comparação entre homens e mulheres jovens com infarto agudo do miocárdio. Arq Bras Cardiol. 2002;79:510-25.

27. Radovanovic D, Erne P, Urban P, Bertel O, Rickli H, Gaspoz JM. Gender differences in management and outcomes in patients with acute coronary syndromes: results on 20290 patients from the AMIS PLUS registry. Heart 2007; 93:1369-75.

28. Rahimtoola SH, Bennett AJ, Grunkemeier GL, Block P, Starr A. Survival at 15 to 18 years after coronary bypass surgery for angina in women. Circulation. 1993;88 (part 2):71-8.

29. Vaccarino V, Abramson J, Veledar E, Weintraub WS. Sex differences in hospital mortality after coronary artery bypass surgery. Evidence for a higher mortality in younger women. Circulation. 2002;105:1176-81.

30. Humphries KH, Gao M, Pu A, Lichtenstein S, Thompson CR. Significant improvement in short-term mortality in women undergoing coronary artery bypass surgery (1991 to 2004). J Am Coll Cardiol. 2007;49:1552-8.

31. Puskas JD, Edwards FH, Pappas PA, O`Brien S, Peterson ED, Kilgo $\mathrm{P}$, et al. Off-pump techniques benefit men and narrow the disparity in mortality after coronary bypass grafting. Ann Thorac Surg. 2007;84:1447-56

32. Kelsey SF, James M, Holubkov AL Holubkov R, Cowley MJ, Detre KM. Results of percutaneous transluminal coronary angioplasty in women. 1985-1986 National Heart, Lung, and Blood Institute's Coronary Angioplasty Registry Circulation. 1993;87:720-7. 
33. Greenberg MA, Mueller HS. Why the excess mortality in women after PTCA? Circulation. 1993;87:1030-1.

34. Jacobs AK, Kelsey SF, Yeh W, Holmes DR Jr, Block PC, Cowley MJ, et al. Documentation of decline in morbidity in women undergoing coronary angioplasty (a report from the 1993-94 NHLBI Percutaneous Transluminal Coronary Angioplasty Registry). National Heart, Lung, and Blood Institute. Am J Cardiol. 1997;80:979-84.

35. Jacobs AK, Kelsey SF, Brooks MM, Faxon DP, Chaitman BR, Bittner V, et al. Better outcome of women compared with men undergoing coronary revascularization: a report from the bypass angioplasty revascularization investigation (BARI). Circulation. 1998;98:1279-85.

36. Vakili BA, Kaplan RC, Brown DL. Sex-based differences in early mortality of patients undergoing primary angioplasty for first acute myocardial infarction. Circulation. 2001;104:3034-8.

37. Watanabe CT, Maynard C, Ritchie JL. Comparison of short-term outcomes following coronary artery stenting in men versus women. Am J Cardiol. 2001;88: 848-52.

38. Da Luz PL, Solimene MC. Terapêutica de reposição hormonal: fim da controvérsia? Rev. Bras. Med. 2003;60:337-40.

39. Effects of estrogen or estrogen/progestin regimens on heart disease risk factors in postmenopausal women. The Postmenopausal Estrogen/ Progestin Interventions (PEPI) Trial. The Writing Group for the PEPI Trial. JAMA. 1995;273:199-208.
40. Hulley S, Grady D, Bush T, Furberg C, Herrington D, Riggs B, et al. Randomized trial of estrogen plus progestin for secondary prevention of coronary heart disease in postmenopausal women. Heart and Estrogen/ progestin Replacement Study (HERS) Research Group. JAMA. 1998;280:605-13

41. Writing Group for the Women's Health Initiative Investigators. Risks and benefits of estrogen plus progestin in healthy postmenopausal women. Principal results from the Women's Health Initiative randomized controlled trial. JAMA. 2002;288:321-33.

42. The Women's Health Initiative Steering Committee. Effects of conjugated equine estrogen in postmenopausal women with hysterectomy. The Women's Health Initiative Randomized Contolled Trial. JAMA. 2004;291:1701-12.

43. Rossouw JE, Prentice RL, Manson JE, Wu LL, Barad D, Barnabei VM, et al. Postmenopausal hormone therapy and risk of cardiovascular disease by age and years since menopause. JAMA. 2007;297:1465-77.

44. Mosca L, Banka CL, Benjamin EJ, Berra K, Bushnell C, Dolor RJ, et al. Evidence-based guidelines for cardiovascular disease prevention in women: 2007 update. J Am Coll Cardiol, 2007;49:1230-50.

45. Collins P, Rosano G, Casey C, Daly C, Gambaccian M, Hadji P, et al. Management of cardiovascular risk in the peri-menopausal woman: a consensus statement of European cardiologists and gynaecologists. Eur Heart J. 2007;28:2028-40. 